\title{
Activated escape of driven systems
}

\author{
M. I. Dykman ${ }^{1}$, I. A. Khovanov ${ }^{2}$, D. G. Luchinsky ${ }^{3,4}$, R. Mannella ${ }^{5,3}$, \\ P. V. E. McClintock ${ }^{3}$, and V. N. Smelyanskiy ${ }^{6}$ \\ ${ }^{1}$ Department of Physics and Astronomy, Michigan State University, East Lansing, MI 48824 \\ ${ }^{2}$ Department of Physics, Saratov State University, Astrakhanskaya St. 83, \\ 410026 Saratov, Russia \\ ${ }^{3}$ Department of Physics, Lancaster University, Lancaster LA1 $4 Y B$, UK \\ ${ }^{4}$ Russian Research Institute for Metrological Service, Ozernaya 46, 119361 Moscow, Russia \\ ${ }^{5}$ Dipartimento di Fisica, Università di Pisa and and INFM UdR Pisa, \\ Piazza Torricelli 2, 56100 Pisa, Italy \\ ${ }^{6}$ Caelum Research Co., NASA Ames Research Center, MS 269-2, Moffett Field, CA 94035-1000
}

\begin{abstract}
.
The problem of activated escape in a far-from-equilibrium system is analysed theoretically, numerically and by analogue electronic experiments. The process is shown to occur via optimal fluctuations that are well described, under diverse conditions, in terms of a new physical quantity, the logarithmic susceptibility (LS).
\end{abstract}

\section{INTRODUCTION}

The question of how a system will respond to an external field is one of the fundamental problems of physics. Where the system is noisy and metastable, and the field is strong and of high frequency compared to reciprocal characteristic times of the system, we have the celebrated nonadiabatic escape scenario - which for many years represented a major unsolved problem in the physics of noise-driven systems. We discuss below how it can now be addressed by use of the logarithmic susceptibility, and we identify two of the (many) related problems remaining to be tackled.

When the driving force is sufficiently weak, the response is proportional to the field amplitude and can be analyzed using linear response theory [1]. The analysis of nonlinear response is more complicated, but can often be extremely interesting and important, e.g. for nucleation in phase transitions. The mechanism responsible can readily be understood for low frequency (adiabatically slow) driving where the system remains in quasi-equilibrium under the instantaneous value of the driving force. For systems in thermal equilibrium, the probabilities of escape are given by the activation law, $W \propto \exp [-R / k T]$ where $R$ is the activation energy of escape. The driving force modulates the value of $R$ quasistatically and, even where the 
modulation amplitude is small compared to $R$, it may still substantially exceed $k T$, in which case $W$ will be changed exponentially strongly. We emphasize that the change of the activation energy is linear in the field amplitude.

For higher field frequencies, where the driving becomes nonadiabatic, one might think that the change of the escape activation energy $R$ will depend on the intensity $I$ of the driving field rather than just be linear in the field amplitude $A \propto I^{1 / 2}$. In this case, where the system is away from thermal equilibrium, there are no known universal relationships from which the escape probabilities can be obtained [2]: much effort has been put into solving the problem, in diverse contexts, and numerical results have been obtained for specific models (see e.g. $[3,4]$ ).

Recent theoretical results $[5,6]$ show, quite counterintuitively, that for highfrequency driving the change of $R$ should still be proportional to the field amplitude, i.e., $\ln W$ should be linear in $A$. The proportionality coefficient was called the logarithmic susceptibility (LS). Just like the conventional linear susceptibility, the LS relates the response of the system in the presence of external driving to its dynamics in thermal equilibrium in the absence of the driving field.

\section{THE LOGARITHMIC SUSCEPTIBILITY}

The idea underlying the theory of the LS $[5,6]$ is that, although the motion of the fluctuating system is random, in a large rare fluctuation from a metastable state to a remote state, or in a fluctuation resulting in escape, the system is most likely to move along a particular trajectory known as the optimal path (see [7-11] and references therein). The effect of a comparatively weak field on the escape probability can therefore be understood in terms of the work that the field does on the system as it moves along the optimal path. One may expect this work to be related to the field-induced change in the activation energy $R$ for the corresponding large fluctuation. This change is linear in the field, provided that the field-induced change of the optimal path itself is negligible. It follows from these arguments that in the case of periodic driving $F(t)=\sum_{k} F_{k} \exp (i k \Omega t)$, the leading-order correction $\delta R$ to the activation energy of escape is

$$
\begin{aligned}
& \delta R=\min _{t_{c}} \delta R\left(t_{c}\right), \delta R\left(t_{c}\right)=\sum_{k} F_{k} \tilde{\chi}(k \Omega) e^{i k \Omega t_{c}}, \\
& \tilde{\chi}(\Omega)=-\int_{-\infty}^{\infty} d t \dot{q}^{(0)}(t) e^{i \Omega t}, \dot{q}^{(0)}=U^{\prime}\left(q^{(0)}\right) .
\end{aligned}
$$

Here, $\tilde{\chi}(\Omega)$ is the LS for escape. It is given $[5,6]$ by the Fourier transform of the velocity along the most probable escape path $q^{(0)}(t)$ in the absence of driving $(F(t)=0)$. The path $q^{(0)}(t)$ is an instanton [12]: it starts for $t \rightarrow-\infty$ at the metastable minimum $q_{s}$ of the potential $U(q)$ and goes for $t \rightarrow \infty$ to the top $q_{u}$ of the potential barrier over which the particle escapes. The minimization over $t_{c}$ corresponds to choosing the position of the center of the instanton so as to maximize the work the field $F(t)$ does on the system along the escape path $q^{(0)}\left(t-t_{c}\right)$. We 


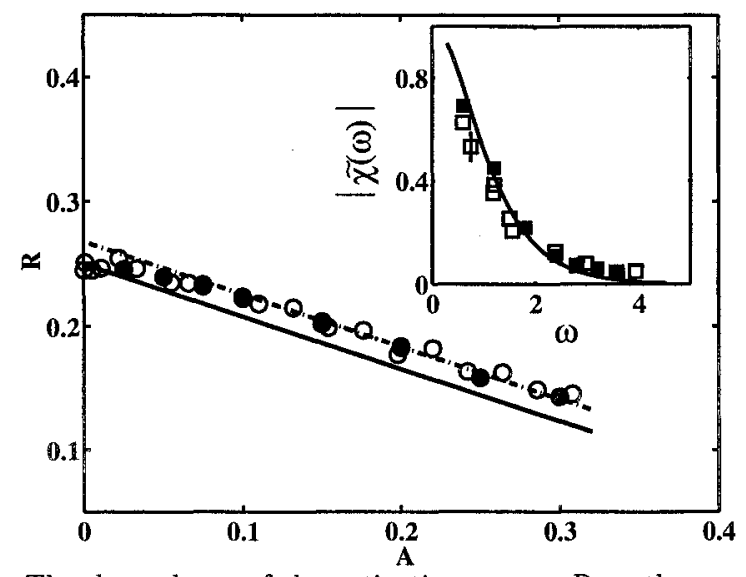

FIGURE 1. The dependence of the activation energy $R$ on the amplitude $A$ of the harmonic driving force $F(t)=A \cos (1.2 t)$ as determined by electronic experiment (filled circles), numerical simulations (open circles) and analytic calculation (solid line) based on (1) for an overdamped Duffing oscillator $U(q)=-q^{2} / 2+q^{4} / 4$; the dash-dot line, drawn parallel to the full curve, is a guide to the eye. The inset shows the absolute value of the LS of the system $|\tilde{\chi}(\Omega)|(1)$ measured (filled and open squares for experiment and numerical simulation, respectively) and calculated (full curve) as a function of frequency $\Omega$ using (2) with $\tilde{\chi}(0)=-1$ and $\tau_{p}=\pi / 2, M=-(1+i)(\pi \omega)^{1 / 2}$ in (2).

note that, for Markov systems in thermal equilibrium, optimal fluctuational paths are the time-reversed relaxational paths in the absence of noise [13-15].

Unlike the standard linear susceptibility [1] which, on causality arguments, is given by a Fourier integral over time from 0 to $\infty$, the LS $\tilde{\chi}(\Omega)$ is given by an integral from $-\infty$ to $\infty$. The analytic properties of $\tilde{\chi}(\Omega)$ therefore differ from those of the standard susceptibility, and in particular their high-frequency asymptotics are qualitatively different. The standard susceptibility for damped dynamical systems decays as a power law for large $\Omega$ (e.g., as $1 /\left[U^{\prime \prime}\left(q_{s}\right)-i \Omega\right]$, for the model of damped Duffing oscillator). In contrast, from (1) the LS decreases exponentially fast,

$$
\tilde{\chi}(\Omega)=M e^{-|\Omega| \tau_{p}}, \quad \tau_{p}=\min \left|\operatorname{Im} \int d q / U^{\prime}(q)\right| .
$$

Here, the integral is taken from any point in the interval $\left(q_{s}, q_{u}\right)$ to the (complex) position $q_{p}$ of the appropriate singularity of $U^{\prime}(q)$. Note that $\dot{q}^{(0)}\left(t-t_{c}\right)$ for given real $t_{c}$ has a pole or a branching point at $\operatorname{Im} t=\tau_{p}$. The prefactor $M$ depends on the form of $U(q)$ near $q_{p}$ and can be obtained in a standard way. In particular, for a polynomial potential $\left(\left|q_{p}\right| \rightarrow \infty\right)$ with $U(q)=C q^{n} / n$ for $|q| \rightarrow \infty$, we have

$$
|M|=2 \pi|\Omega / C|^{\nu}|\nu|^{\nu+1} / \nu !, \nu=1 /(n-2) .
$$

This expression applies also for finite $\left|q_{p}\right|$, with $U(q) \approx C / \mu\left(q-q_{p}\right)^{\mu}$ for $q \rightarrow q_{p}$, if $n$ in (3) is replaced by $-\mu$ : note that $|M|$ then decreases with increasing $\Omega$. 
For weak damping, $|\tilde{\chi}(\Omega)|$ displays sharp peaks, and may have a multiply-peaked structure [5]. It can be understood if one writes the velocity on the MPEP as

$$
\begin{aligned}
& \dot{q}^{(0)}(t) \equiv P(t)=\operatorname{Re} \sum_{n>0} P_{n}(E(t)) \exp [-i n \phi(t)], \\
& \dot{E} \approx 2 \Gamma \omega(E) I(E), \quad \dot{\phi} \approx \omega(E),
\end{aligned}
$$

where $\Gamma$ is the damping of the oscillator, $\omega(E)$ is the eigenfrequency of the vibrations that have energy $E$ in the absence of dissipation, $I(E)$ is the action for these vibrations, and $P_{n}(E)$ is the amplitude of the $n$th overtone of the momentum $P$; terms $\propto \Gamma$ in the equation for the phase, and fast-oscillating terms in that for the energy, have been dropped.

Resonant contributions to $|\tilde{\chi}(\omega)|$ come from the vibrations of energy $E_{n}(\omega)$ for which $n \omega\left(E_{n}\right)=\Omega$. In the range of $\Omega$ where the contribution from an overtone with a given $n$ is much larger than from other overtones, we may approximate $|\tilde{\chi}(\omega)|$ by the function $\left|\tilde{\chi}_{n}(\omega)\right|$ calculated from Eqs. (1) taking into account in (4) the term with one $n$ only. Evaluating the integral over time in (1) by the steepest descent method, we obtain

$$
\left|\tilde{\chi}_{n}(\Omega)\right|=\left[\left|P_{n}(E)\right|\left|\frac{2 \Gamma n I(E)}{\pi} \frac{d \omega^{2}}{d E}\right|^{-1 / 2}\right]_{E=E_{n}} .
$$

To test these predictions, we have built an analog electronic model [16] of the overdamped motion of a Brownian particle in the double-well Duffing potential. We drive it with zero-mean quasi-white Gaussian noise from a shift-register noise generator, digitize the response $q(t)$, and analyse it with a digital data processor. We have also carried out a complementary digital simulation [17]. Numerical simulations in the case of small damping are currently in progress: preliminary results indicate a resonant behaviour of the LS. The analogue and digital measurements of $R$ involved noise intensities in the ranges $D=0.028-0.036$ and $D=0.020-0.028$ respectively; the lowest (real time [16]) driving frequency used was $460 \mathrm{~Hz}$. The results are plotted in Fig. 1. The major observation is that, as expected, $R$ is indeed linear in the force amplitude $(R=1 / 4$ for $A=0)$. The slope yields the absolute value of the LS. Its frequency dependence, a fundamental characteristic of the original equilibrium system, is compared with the theoretical predictions (2) in the inset of Fig. 1.

\section{OPEN PROBLEMS}

There are, of course, numerous related problems of obvious importance that can now be addressed through use of our new theoretical, experimental and numerical techniques. Two that we are considering are: noisy escape from a strange attractor; and large fluctuations in systems that have limit cycles. 

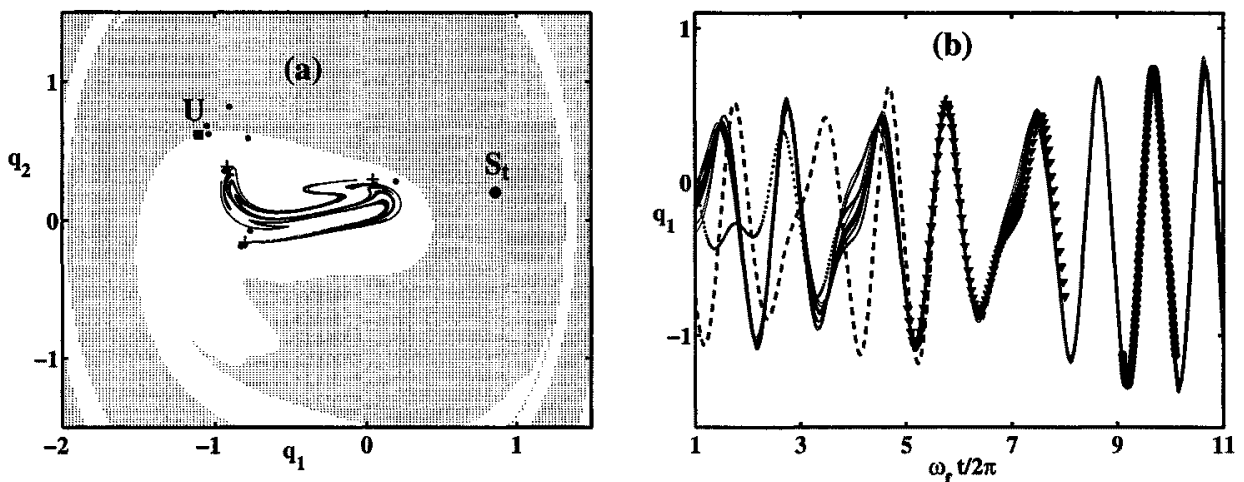

FIGURE 2. (a) Basins of attraction of the quasi-attractor $(Q A)$ and stable limit cycle $\left(S_{t}\right.$ shown by the large black dot) for (6) are shown as white and shaded regions in Poincaré section for $\omega_{f} t=0.6 \pi, \omega_{0}=0.579, \beta=1, \gamma=1, \Gamma=0.05, \omega_{f}=0.95, h=0.13$. The intersections of an actual fluctuational escape trajectory with this Poincaré section are shown by the small black dots. The unstable limit cycle $(U)$ at the boundary of the $Q A$ is shown by the filled square. The saddle limit cycle which can be considered as a boundary of the $Q A$ itself is shown as crosses. (b) Three optimal escape paths found in numerical simulations with $T=0.002$ using the method of optimal trajectories are shown by dotted, full and dashed lines; their relative probabilities are $9.5: 4: 1$ respectively. One period of the unstable saddle cycle $(S 3)$ and unstable limit cycle $(U)$ are shown by triangles and thick black dots respectively; 15 measured fluctuational escape trajectories corresponding to the middle-probability optimal path are shown by thin full lines.

Preliminary experiments on the first of these indicate that escape over the basin boundary of a strange attractor involves only a few periodic limit cycles that can be found numerically: Fig. 2 shows results for a periodically-driven weakly damped oscillator

$$
\begin{aligned}
& \dot{\mathbf{q}}=\mathbf{K}(\mathbf{q}, t)+\mathbf{f}(t), \quad \mathbf{K}=\left\{q_{2},-2 \Gamma q_{2}-\omega_{0}^{2} q_{1}-\beta q_{1}^{2}-\gamma q_{1}^{3}+h \cos \left(\omega_{f} t\right)\right\} \\
& \mathbf{f}(t)=\{0, \xi(t)\}, \quad<\xi(t)>=0, \quad<\xi(t) \xi(0)>=4 \Gamma k T \delta(t), \quad \Gamma \ll \omega_{f},
\end{aligned}
$$

The oscillator was monostable $\left(\gamma>0, \beta^{2}<4 \gamma \omega_{0}^{2}\right)$ and the energy dependence of its eigenfrequency was nonmonotonic $\left(\frac{\beta^{2}}{\gamma \omega_{0}^{2}}>\frac{9}{10}\right)$.

In the case of systems with limit cycles, it has been shown [18] that, in the vicinity of the unstable focus inside the cycle, the flow field of optimal paths generically displays a pattern of singularities. It contains a self-similar switching line that separates areas to which the system arrives along optimal paths of topologically different types. We have already been able to show how this topological pattern emerges as one moves away from the detailed balance condition assumed in a large body of previous work. 


\section{ACKNOWLEDGEMENTS}

The work was supported by the Engineering and Physical Sciences Research Council (UK) under grants Nos. GR/L01978, GR/L09646, GR/L38875 and GR/L99562, by INTAS under grants Nos. INTAS-96-0305 and INTAS-97-0574, by the Royal Society of London, and by the National Science Foundation (US) under grant No. PHY-9722057, and by the Center for Fundamental Material Research at MSU.

\section{REFERENCES}

1. Landau, L.D. and Lifshitz, E.M., Statistical Physics (Pergamon: New York, 1980).

2. Landauer, R., J. Stat. Phys. 13, 1-4 (1975); J. Stat. Phys. 53, 233-248 (1988).

3. Jung, P., Phys. Rep. 234, 175-295 (1993).

4. Reichl, L.E. and Kim, S., Phys. Rev. E 53, 3088-3094 (1996).

5. Dykman, M.I., Rabitz, H., Smelyanskiy, V.N. and Vugmeister, B.E., Phys. Rev. Lett. 79, 1178-1181 (1997).

6. Smelyanskiy, V.N., Dykman, M.I., Rabitz, H. and Vugmeister, B.E., Phys. Rev. Lett. 79, 3113-3116 (1997).

7. Freidlin, M.I. and Wentzel, A.D., Random Perturbations in Dynamical Systems (Springer, New-York, 1984).

8. Dykman, M.I. and Krivoglaz, M.A., in Soviet Physics Reviews, edited by I.M. Khalatnikov (Harwood Academic Publishers, New York, 1984), Vol. 5, pp. 265-442; Dykman, M.I., Phys. Rev. A 42, 2020-2029 (1990).

9. Graham, R. and Tél, T., Phys. Rev. Lett. 52, 9-12 (1984); Graham, R. and Tél, T., Phys. Rev. A 31, 1109-1122 (1985); Graham, R., in Noise in Nonlinear Dynamical Systems, edited by F. Moss and P. V. E. McClintock (Cambridge University Press, Cambridge, 1989), Vol. 1, pp 225-278.

10. Bray, A.J. and McKane, A.J., Phys. Rev. Lett. 62, 493-496 (1989).

11. Maier, R.S. and Stein, D.L., J. Stat. Phys. 83, 291-357 (1996).

12. Schulman, L.S., Techniques and Applications of Path Integration (Wiley, New York 1981).

13. Onsager, L. and Machlup, S., Phys. Rev. 91, 1505-1512 (1953).

14. See Marder, M., Phys. Rev. Lett. 74, 4547-4550 (1995) and references therein.

15. Luchinsky, D.G., Maier, R.S., Mannella, R., McClintock, P.V.E. and Stein, D.L., Phys. Rev. Lett. 79, 3109-3112 (1997).

16. Luchinsky, D.G., McClintock, P.V.E. and Dykman, M.I., Rep. Prog. Phys. 61, 889997 (1998).

17. Mannella, R., in Supercomputation in Nonlinear and Disordered Systems, eds. L.Vázquez, F. Tirando, and I. Martin (World Scientific, Singapore, 1997), pp. 100130.

18. Dykman, M.I., Smelyanskiy, V.N., Maier, R. and Silverstein, M., J. Phys. Chem. 100, 19197-19209 (1996); Smelyanskiy, V.N., Dykman, M.I. and Maier, R.S., Phys. Rev. E 55, 2369-2391 (1997). 